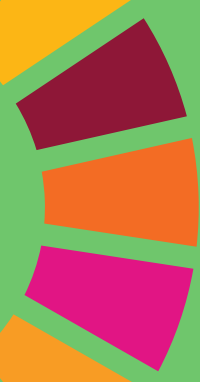

\title{
ANÁLISIS CRÍTICO DE LA RENTA BASICA: COSTES E INCENTIVOS. APLICACIÓN AL CASO ESPAÑOL'
}

\section{A CRITICAL APPROACH TO BASIC INCOME: COSTS AND INCENTIVES. AN APPROXIMATION TO THE CASE OF SPAIN}

\author{
José María Casado \\ Economista \\ Miguel Sebastián \\ ICAE, Universidad Complutense de Madrid
}

\section{RESUMEN}

Este trabajo estima los costes de la renta básica y analiza los problemas de incentivos que puede generar, para concluir que existe una relación inversa entre ambos. Cuanto más incondicional es la renta básica menos problemas de incentivos provoca, pero más costosa es desde un punto de vista financiero. Se estima, para el caso español, su posible coste utilizando tanto un enfoque macroeconómico como uno con micro datos. El elevado coste de aplicar la renta básica universal hace necesario limitar el número de perceptores para que sea posible su implementación, pero esto podría provocar importantes problemas de incentivos a la oferta de trabajo y a la acumulación de capital humano. También se discuten las posibles implicaciones en materia de desigualdad.

Palabras clave: Renta básica, incentivos, costes, condicionalidad, microdatos, desigualdad. 


\section{ABSTRACT:}

This paper estimates the costs of basic income and analyses its incentives to conclude that there is an inverse relationship between them. The more unconditional the basic income is, the less incentive problems will arise, but it will be more costly. We approximate its costs to the case of Spain, both using a macroeconomic approach and one with micro data. The high cost of the universal basic income implementation requires to limit the number of recipients by income or labour status and, therefore, labour supply and human capital incentive problems could be materialized. Implications for inequality are also discussed.

Keywords: Basic income, incentives, costs, conditionality, micro data, inequality.

José María Casado: Doctor por la London School of Economics, Master en Economía por University College London, Diploma de Estudios Avanzados por la Universidad de Zaragoza y Licenciado en Economía por la Universidad de Extremadura. En la actualidad es Coordinador Adjunto del Spending Review, Jefe de Proyecto de Beneficios Fiscales de la AIReF y Economista Titulado del Banco de España desde el año 2009. Ha publicado diversos artículos en revistas internacionales en el ámbito de la microeconometría sobre evaluación de políticas públicas, microsimulación fiscal, desigualdad y comportamiento del consumidor.

Miguel Sebastián: Licenciado en Económicas por la Universidad Complutense de Madrid, PH.D. in Economics, University of Minnesota. Profesor Titular de Análisis Económico de la Universidad Complutense. Ha publicado numerosos articulos y varios libros, el más reciente La Falsa Bonanza. Ha trabajado en el Servicio de Estudios del Banco de España y en el Ministerio de Economía y Hacienda. Ha sido Director General de InterMoney, así como Economista Jefe y Subdirector General de BBV y de BBVA. Director de la Oficina Económica del Presidente del Gobierno y Ministro de Industria, Turismo y Comercio durante toda la IX Legislatura. En la actualidad es Consejero dominical de Indra, en representación de la SEPI.

\section{INTRODUCCIÓN}

Aunque el origen del concepto de "renta básica universal" se remonta a Thomas Paine, un político liberal del siglo XVIII y uno de los padres de la independencia de los EE.UU., el término ha cobrado relevancia recientemente, al haberse incorporado como propuesta de política económica en muchos países desarrollados². Pese a su origen liberal, la renta básica universal es ahora una bandera de la izquierda en muchos de esos países. Y lo es como un intento de respuesta a varias cuestiones, algunas de ellas indudables y otras más discutibles: (i) el aumento de la desigualdad de la renta dentro de los países industrializados, (ii) el aumento de la población en riesgo de pobreza en algunas economías (iii), la caída del peso de las rentas salariales sobre el PIB, y el aumento secular de las rentas del capital, cuya propiedad se encuentra en pocas manos, (iv) el impacto de la robotización sobre el empleo menos cualificado, que sería condenado a mantenerse fuera del mercado de trabajo de forma permanente y $(v)$ como una forma de reconocer socialmente el trabajo doméstico y de cuidado de los niños, de los dependientes y de los mayores por parte de un porcentaje creciente de la población, sobre todo en los países desarrollados.

\footnotetext{
${ }^{1}$ Los análisis y las opiniones expresadas en este artículo son responsabilidad de los autores y en ningún caso deben ser atribuidas al Banco de España, al Eurosistema o a la AIReF.

2 Un buen resumen de todas las experiencias de renta básica, bajo diferentes versiones, se encuentra en Torres (2019)
} 
A principios de los años 80 del siglo XX, en una situación de fuerte aumento del paro estructural, hubo un intento en Holanda de implantar un "subsidio universal" por parte de uno de los sindicatos. Pero la medida no fue aceptada por la izquierda, porque ponía en riesgo el Estado del Bienestar, y fue abandonada. Pese a este fracaso, en 1985, en la vecina Bélgica, el Colectivo Charles Fourier ${ }^{3}$, formado por Defeyt, van Parijs y Boulanger, planteó "el subsidio universal" sustitutivo de todos los subsidios, becas y prestaciones sociales, acompañado de una profunda reforma del mercado de trabajo, con la abolición del salario mínimo, la jornada máxima de trabajo o la edad de jubilación, y que fue el germen de la Renta Básica Universal (RBU) contemporánea. A partir de los debates generados por esta propuesta en 1986 se creó la Red Europea de Renta Básica, conocida por sus siglas en inglés BIEN (Basic Income European Network), que en 2004 se globaliza, utilizando las mismas siglas, bajo la red Basic Income Earth Network. La red opera ya en 30 países. También en España funciona desde hace tiempo la RRB (Red Renta Básica).

Con la crisis financiera global y el aumento generalizado del desempleo y de la desigualdad, la hasta entonces propuesta más bien teórica o filosófica comienza a convertirse en una opción real de política económica.

En junio de 2016 se planteó en Suiza la primera consulta pública relacionada con la implantación de alguna forma de subsidio universal. La población rechazó en un referéndum de forma abrumadora (un $78 \%$ de los votos) la implantación de una renta básica de 2.500 francos suizos (unos 2.200 euros), aunque no fuera compatible con otras fuentes de renta. Pese a ese rechazo, la idea continuó siendo estudiada en Finlandia, en una fase experimental en 2017. La experiencia se llevó a cabo en una muestra de población de 2.000 personas en paro de larga duración, no voluntarias y seleccionadas al azar. Durante un período de dos años, los participantes recibieron un ingreso básico mensual de 560 euros sin tener que cumplir con ningún requisito burocrático para poder obtener la ayuda mensual. El objetivo era saber si ese mínimo garantizado de protección, los ayudaría a encontrar trabajo y les daría un apoyo, en caso de que se vieran obligados a tomar empleos esporádicos. Lo cierto es que el experimento fracasó porque no se consiguió aumentar el empleo. Trabajaron prácticamente las mismas horas y ganaron lo mismo, exceptuando los ingresos de la renta básica, que otro colectivo de características similares, aunque los participantes reconocieron sentirse más felices y menos estresados. Aunque el experimento se consideró como un fracaso en sus objetivos y se suspendió al acabar 2018, los defensores del proyecto insisten en que una renta básica, sin condiciones de ningún tipo, evita que los receptores de ayudas rechacen empleos por temor a superar el nivel de ingresos mínimos para recibir subsidios. Y que al no tener que ocuparse de la burocracia con los servicios sociales podrían dedicar ese tiempo a buscar empleo de forma más eficaz.

En otros países europeos también ha habido iniciativas parecidas. La renta básica fue una de las propuestas estrella del Partido Socialista Francés en 2017, pero el partido tuvo un muy desfavorable resultado en las elecciones (un 6\%), aunque probablemente explicado por el desgaste del último Presidente socialista, François Hollande, que fue el único caso que no se presentó a una reelección, dada su impopularidad. El líder laborista británico, Jeremy Corbyn, también ha anunciado que "lo estudiará" si gana las elecciones. El creador y CEO de Facebook, Mark Zuckerberg, recomendó, en el discurso de ceremonia de graduación de Harvard, que se "explore" sobre la renta básica universal. Recientemente el aspirante demócrata a las primarias para las elecciones presidenciales en EE.UU. en 2020, Andrew Yang, introdujo en un debate entre los 10 posibles candidatos del Partido Demócrata, su idea de Freedom Dividend o Dividendo de la Libertad, una prestación de $1.000 \$$ mensuales a todos los ciudadanos adultos, con carácter incondicional.

${ }^{3}$ Véase Collectiff Charles Fourier (1985) 
En España, la formación política Podemos ha llevado en sus programas electorales diferentes versiones de la renta básica universal. Y el Partido Socialista Obrero Español aprobó, en su $39^{\circ}$ Congreso Federal de junio de 2017, incluir alguna versión de la renta básica para su futuro programa electoral. En mayo de 2016 los sindicatos mayoritarios, CCOO y UGT, presentaron 700.000 firmas en favor de una Iniciativa Legislativa Popular (ILP) para "poner en marcha un nuevo derecho subjetivo vinculado a la Seguridad Social y financiado a través de los Presupuestos Generales". Esta iniciativa, que fue admitida a trámite en la Comisión de Empleo y Seguridad del Congreso de los Diputados, reabrió el debate sobre la "renta básica", o sus variantes (renta mínima, renta de inserción, etc.). Los sindicatos no pedían exactamente una "renta básica de ciudadanía", sino una renta mínima para desempleados, y no "incondicional", sino vinculada a la búsqueda de empleo o a la participación en programas de inserción laboral. A iniciativa del Grupo Socialista, se tramitó una Proposición de Ley. En febrero de 2018 el Congreso de los Diputados votó en contra de la renta básica universal. 170 votos en contra (PP, Ciudadanos, PNV, UPN y Foro Asturias) contra 168 a favor (PSOE, Unidos Podemos, ERC, Partido Demócrata, Compromís, Bildu, Coalición Canaria y Nueva Canarias). Varios diputados socialistas estuvieron ausentes y uno votó en contra, lo que imposibilitó su aprobación, que hubiera supuesto una ayuda de 426 euros al mes para unos 2,1 millones de potenciales beneficiarios, familias que no tienen ningún ingreso o que se encuentran por debajo del umbral de la pobreza.

\section{EL CONCEPTO DE RENTA BÁSICA}

El debate sobre la Renta Básica Universal (RBU) crea mucha confusión, porque muchos de sus partidarios y de sus detractores mezclan en sus argumentos diferentes conceptos: el de "renta básica universal" (para todos los ciudadanos, por el hecho de serlo), el de renta mínima o renta de inserción (sólo para parados de larga duración, que no tengan o hayan agotado su prestación de desempleo), el "complemento salarial" financiado con recursos públicos (sólo para empleados de rentas bajas) o el impuesto negativo de Friedman (sólo para trabajadores empleados que ganan por debajo de un determinado umbral y que, en su declaración fiscal, recibirían una renta, en lugar de pagar un impuesto).

Entendemos por "renta básica universal” o "incondicional” una percepción económica garantizada por el sector público y a la que tendrían derecho todos los ciudadanos de un país por el hecho de serlo, independientemente de que estén o no en edad de trabajar, de que sean activos o inactivos, o de que estén ocupados o parados.

Generalmente el debate sobre la conveniencia o no de la renta básica se centra en su coste. Ese fue el principal argumento que provocó su rechazo en el referéndum de Suiza, y es el principal argumento que esgrimen sus detractores para oponerse a ella. En nuestra opinión, sin embargo, esta es una visión demasiado estrecha, y la renta básica universal debe ser valorada en función de tres características, que no son independientes, pero que pueden ser tratadas de forma separada:

1.Su coste, tanto a corto como medio y largo plazo.

2.Sus problemas de incentivos.

3.Su impacto sobre la pobreza y la distribución de la renta. 
Como veremos más adelante, los tres conceptos son distintos, pero no son independientes entre sí. Por ejemplo, los posibles incentivos negativos de la RBU podrían incidir en su coste a largo plazo, que serían muy superiores al coste a corto plazo si, por ejemplo, mucha gente decide no trabajar, se desactiva, y se pierde crecimiento económico y recaudación. Otro ejemplo: un elevado coste fiscal a corto plazo podría limitar los recursos disponibles para otras herramientas de lucha contra la pobreza o la desigualdad, empeorando la tercera de las vertientes. Más adelante trataremos estas tres características en profundidad. Pero a continuación queremos mostrar la relación que existe entre costes e incentivos, para señalar el problema de elección social que se le plantea al policy maker.

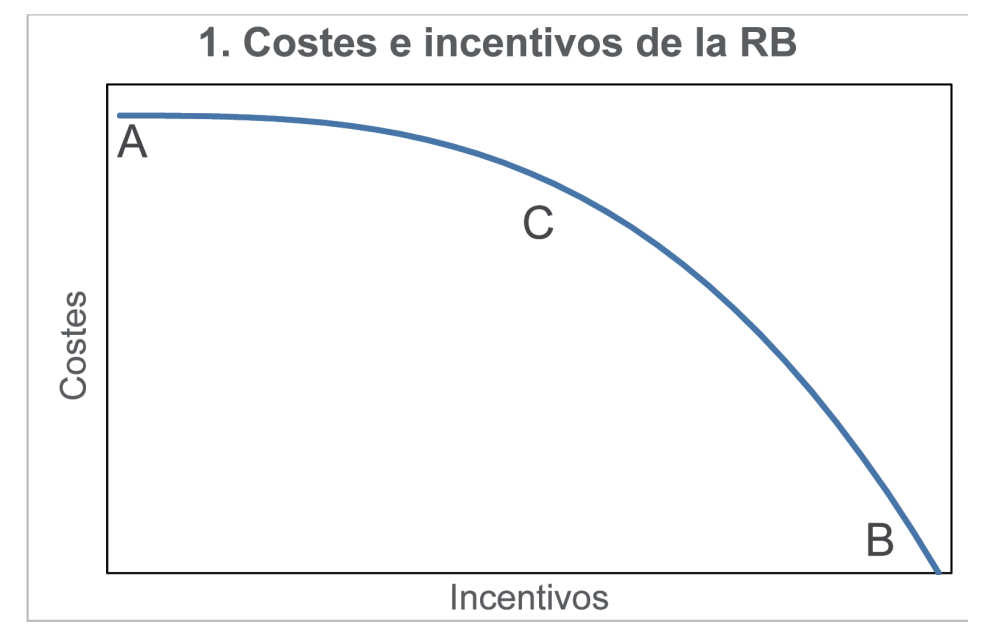

En el Diagrama 1 se presenta una ilustración de este dilema que surge entre costes e incentivos. Cuantos menos problemas de incentivos queramos tener, habrá que pagar un mayor coste de implantación de la RB. Y, viceversa, si queremos tener un coste más reducido, introduciendo condicionalidad o sustitución de partidas por otras ya existentes, el diseño de la RB incurrirá en mayores problemas de incentivos. A continuación, trataremos de explicar este dilema. El punto $A$ es el que corresponde con una situación donde no hay problemas de incentivos, pero en el que el coste de la RB es máximo. Se trataría, por ejemplo, de un sistema en el que todos los ciudadanos reciben, por el hecho de serlo, una misma cuantía fija, independientemente de la situación laboral o personal en que se encuentren. Como la percepción de esa cuantía es incondicional, para cada ciudadano tomado individualmente no existiría ningún incentivo a cambiar su situación de partida. Es decir, si la RB se va a percibir independientemente de si es activo o inactivo, de si está parado u ocupado, o de su nivel de renta, dicha renta no cambiará la decisión que ha llevado a ese individuo a estar en esa situación. Este punto, que en el gráfico lo designamos con la letra A, puede ser asociado estrictamente a la Renta Básica Universal (RBU), es decir, la percepción de una renta generalizada e incondicional. En el otro extremo del diagrama se encuentra el punto B en el que el coste de implantar la RB es muy bajo, pero surgen graves problemas de incentivos. Si el coste es bajo es porque la RB está sustituyendo una buena parte de las rentas percibidas por otros conceptos, o que determinados ciudadanos dejan de ser elegibles para la prestación si no se dan una serie de condiciones. Por ejemplo, un diseño en el que los trabajadores no recibirían la RB por disponer de rentas salariales. Otro caso incluido en este mismo punto del diagrama es aquel en que no perciben ningún ingreso adicional los parados que ya perciban prestación por desempleo. Es decir, o no perciben la RB o la RB sustituiría a dicha prestación. Finalmente, también podríamos considerar en este tramo de la curva en el que la RB no aporta renta adicional, ni coste para el erario público, si los pensionistas que reciban una pensión contributiva la vean sustituida por esta renta básica o simplemente queden excluidos de su percepción por tener ya una renta vital. Es evidente que, en todos estos casos, tomados aisladamente o en su conjunto, habría un coste menor de implantar la RB. Pero también debe quedar claro que con este esquema surgen multitud de incentivos: los trabajadores de rentas más bajas podrían preferir dejar de trabajar y recibir la RB. Es decir, podría haber una reducción de la "oferta de trabajo efectiva", lo que elevaría los costes para las empresas. Los parados podrían perder el 
incentivo a encontrar trabajo, dada esta "prestación indefinida", lo que elevaría la tasa de paro estructural y el desempleo de larga duración. Y los futuros pensionistas podrían tener un incentivo a dejar de cotizar, dado que la RB se percibe como una prestación no contributiva, lo que dañaría la sostenibilidad del sistema de pensiones.

$\mathrm{Y}$, de vuelta al diagrama, entre medias de los dos extremos se encuentra un punto como el C, donde el coste de implantar la RB es significativo, aunque no es tan elevado como en el caso universal y en el que existen algunos problemas de incentivos, aunque no tan elevados como en el caso en el que los perceptores de rentas quedan excluidos de este subsidio adicional.

Cuál será el punto elegido por los responsables políticos dependerá de las preferencias sociales, de la restricción de recursos y del grado de altruismo, entendido éste como la aceptación de una buena parte de la sociedad de financiar con sus impuestos el establecimiento de esta renta garantizada, aunque ellos no vayan a ser los perceptores. Las sociedades más ricas (por ejemplo, Noruega) o más saneadas fiscalmente podrán optar por un punto como el $\mathrm{A}$, al entender que todos se benefician de ese esfuerzo fiscal y no se generan problemas de incentivos. Las sociedades que tengan un mayor problema de recursos estarán tentadas a optar por un punto como el B. Nuestro punto, sin embargo, es que, aunque se trate de una solución más factible a corto plazo, presenta problemas de sostenibilidad a largo plazo. Y ello por varios motivos. En primer lugar, porque los incentivos negativos podrían operar a largo plazo, produciendo una merma de recursos para el Estado del Bienestar en su conjunto. En segundo lugar, porque el tratamiento diferencial que reciben unos ciudadanos frente a otros podría generar rechazo social a largo plazo, sobre todo en sociedades con un grado de altruismo insuficiente. Por todo ello, creemos que una solución como la B será factible pero inestable, mientras que una solución como la A será costosa pero estable.

En los países donde se implante la RB probablemente se opte por una opción intermedia. Pero, en aras de garantizar su estabilidad, a partir de un sistema inicial, por ejemplo, el C, si se descubre que el coste es asumible los sucesivos gobiernos deberían tratar de moverse a lo largo de la curva hacia arriba, para reducir el problema de incentivos y garantizar la sostenibilidad a largo plazo. Por el contrario, si el coste es inasumible, los sucesivos gobiernos buscarán moverse en la dirección contraria, hacia abajo a lo largo de la curva. Se contendrá el gasto con medidas de condicionalidad o sustitución, pero ello hará que empeore el problema de incentivos y que aumente el rechazo social de los que sientan algún tipo de agravio comparativo.

Aunque ya lo hemos mencionado anteriormente, estos problemas de incentivos se traducen, en general en una menor eficiencia en la asignación de recursos y en un menor crecimiento a largo plazo. Ello afectará a la recaudación fiscal del futuro, por lo que, en realidad, la curva representada en el Diagrama 1 podría también interpretarse como un dilema entre los costes a corto plazo y los costes a largo plazo derivados de la implantación de la RB. Sin embargo, este es un enfoque mucho más complejo de abordar, pues es muy difícil modelizar. Como la evaluación de los costes a largo plazo es compleja, preferimos dejar el dilema entre "costes" e "incentivos", pero siendo conscientes del impacto a largo plazo de los diferentes desincentivos y su impacto sobre la recaudación estructural. En el resto del papel abordaremos por separado las tres características anteriormente expuestas, empezando por los costes, siguiendo por los incentivos y terminando por una reflexión sobre el posible impacto sobre la pobreza y la desigualdad, que son los objetivos finales por los que se implantaría algún esquema de renta básica, aplicado todo ello al caso de la economía española. 


\section{EL COSTE DE LA RENTA BÁSICA}

Hablaremos aquí de varias estimaciones de las diferentes modalidades de renta básica descritas en el Diagrama 1, empezando por la Renta Básica Universal (RBU). Cuando hablamos de RBU nos vamos a referir, por tanto, a una situación como la ilustrada en el punto A de dicho diagrama, es decir, una renta incondicional y universal, independiente de las características laborales o personales de cada receptor, y en la que no se incurre en problemas de incentivos, pero sí en unos elevados costes. Dentro de dichos costes habría que incluir, los "costes de entrada", es decir, de implantación de la medida a corto plazo. Asimismo, habría que considerar los "costes de salida", es decir, los potenciales costes políticos y sociales de echar marcha atrás en la RBU, una vez implantada en un país y haberse demostrado inviable a medio plazo. Por simplicidad, nos referiremos solamente al primero de los costes, aunque ya hemos mencionado antes los posibles problemas de sostenibilidad de los diferentes modelos de renta básica.

¿Cuál puede ser el "coste de entrada" de la RBU? Evidentemente ese coste va a depender de dos factores: la cuantía de la RBU y el número de perceptores anuales.

Tomemos el caso de España. En la Tabla 1 resumimos el coste de implantar una renta básica universal de 500 euros al mes, unos 6.000 euros al año para el total de la Población estimada por el INE para el 1 de enero de 2019, es decir, unos 47 millones de personas.

\begin{tabular}{|c|c|}
\hline \multicolumn{2}{|c|}{ 1. RENTA BÁSICA UNIVERSAL PURA } \\
\hline Población ESPAÑA (miles) & 46935 \\
\hline Renta básica anual pura (en miles) & 6 \\
\hline PIB nominal (en millones) & 1200000 \\
\hline COSTE (en millones de euros) & 281610 \\
\hline COSTE (en \% PIB) & 23,5 \\
\hline
\end{tabular}

Fuente: elaboración propia a partir de INE. Población al comienzo de 2019

El coste de esta medida sería de unos 280.000 millones de euros al año, lo que supondría casi un $24 \%$ del PIB. Su financiación, por tanto, equivaldría a elevar el ratio de deuda en 24 puntos del PIB cada año, en caso de que no se quiera financiar con impuestos, o la presión fiscal en un $60 \%$ de forma permanente, o una combinación intermedia entre ambas opciones. Se trata, por tanto, de un coste muy elevado que, con casi toda seguridad, no podría ser asumido por ningún gobierno o por los mercados financieros, en caso de ser financiado con deuda. El lector pensará que el crecimiento del PIB a largo plazo será superior al de la población, que probablemente decrezca en las próximas décadas como resultado del envejecimiento demográfico. Ello haría que el ratio sobre PIB disminuyera tendencialmente. EI argumento es algo dudoso, porque también habría presiones para elevar la cuantía de dicha RBU, y no sólo por el IPC, a medida que aumente la renta per cápita. Además, el propio envejecimiento demográfico, dado nuestro sistema de pensiones de reparto, va a obligar hasta 2040-50 a disponer de un colchón adicional para acomodar aumentos de deuda pública como consecuencia de atender a la sostenibilidad de dicho sistema. 
Por todos estos argumentos, habrá que considerar un recorte del gasto en la RBU. Dado que la cuantía (500 euros al mes) está bastante ajustada ${ }^{4}$, la única forma de reducir el coste es a través del número de perceptores. Pero, ¿cómo se pueden hacer dichos recortes manteniendo su carácter universal? Una posibilidad, recogida en la Tabla 2, es la de excluir a los menores de 16 años, que se supone que están a cargo de los progenitores. Es cierto que se trata de ciudadanos como todos los demás, por lo que, en principio, deberían recibir una renta que tiene un carácter "universal". Pero tampoco con esa edad tienen derecho a voto, ni al trabajo, ni a conducir un vehículo, como tampoco responden penalmente de sus posibles delitos. Además, al vivir en el mismo hogar, recibirían parte de esa prestación a través de sus padres. Desde un punto de vista de los incentivos, la primera versión universal en la que los jóvenes estaban incluidos en la lista de perceptores, Tabla 1, equivaldría a entregar un "cheque bebé" por importe de 6.000 euros anuales durante 16 años, es decir, unos 96.000 euros por hijo. Se trataría de una política de impulso a la natalidad sin duda muy costosa. Por ello, lo razonable es la exclusión de los menores. El propio Andrew Yang, del Partido Demócrata de EE.UU., propone el "dividendo por la libertad" sólo para adultos.

¿Cuál sería el coste concreto de excluir de la RBU a los jóvenes en España? El resultado se presenta en la Tabla 2. En nuestro país hay unos 7,5 millones de jóvenes menores de 16 años $^{5}$, por lo que excluirles equivale a que los perceptores de la RBU fueran unos 39,2 millones de personas. El coste total de esta RBU ajustada por edad sería de unos 235.000 millones de euros al año. Es decir, un ahorro de unos 46.000 millones de euros por año. En términos de PIB estamos hablando de una factura de casi un $20 \%$ del PIB, con un ahorro de casi 4 puntos del PIB (3,9\%)

\begin{tabular}{|l|r|}
\hline 2. RENTA BÁSICA UNIVERSAL ajuste (a) \\
\hline Población ESPAÑA ADULTOS (miles) \\
\hline Renta básica anual pura (en miles) & 39213 \\
\hline PIB nominal (en millones) & 6 \\
\hline COSTE (en millones de euros) & 1200000 \\
\hline COSTE (en \% PIB) & 235278 \\
\hline
\end{tabular}

Fuente: elaboración propia a partir de INE. Población al comienzo de 2019

Se trata de un importe significativamente menor, pero, aun así, inasumible. Se podría pensar en ajustes adicionales sobre esta partida basada en la edad, por ejemplo, excluir a los jóvenes de más de 25 años que continúen viviendo en casa de sus padres. Si no se les considera elegibles hasta que se vayan de casa, se estaría, además de recortar el coste de la RBU, incentivando su emancipación. En España, según el INE ${ }^{6}$ un $53 \%$ de los jóvenes de 25 a 29 años vivía con sus padres al terminar 2018.

Otra alternativa, más polémica si cabe, sería la de excluir como posibles perceptores a los extranjeros residentes en España. Esta exclusión es más difícil de justificar, porque se trata de personas que, en una buena parte, contribuyen con su trabajo y sus impuestos al mantenimiento del Estado del Bienestar. El argumento económico para justificar esa exclusión sería la de evitar un "efecto llamada", es decir, un aluvión

\footnotetext{
${ }^{4}$ Recuérdese que el salario mínimo se ha fijado este año en 900 euros/mes, y probablemente se eleve más en los próximos años.

${ }^{5}$ Ver INE (2019b): "Encuesta de Población Activa"

${ }^{6}$ Ver INE (2019a): "Encuesta Continua de Hogares".
} 
de llegadas de inmigrantes, por ejemplo, de los países de la UE con los que existen acuerdos de libertad de movimientos, en busca del cobro de la prestación universal. La estimación del coste de la RBU en este caso se presenta en la Tabla 3. Como en España hay aproximadamente 4,8 millones de extranjeros, excluirles como receptores de la RBU supondría en comparación con la Tabla 1, un ahorro de casi 30.000 millones de euros, un $2,4 \%$ del PIB. Se trata, en cualquier caso, de un coste inasumible, tanto en términos de emisiones anuales de deuda, como de un aumento permanente de la presión fiscal.

\begin{tabular}{|l|r|}
\hline 3. RENTA BÁSICA UNIVERSAL ajuste (b) \\
\hline Población ESPAÑOLES (miles) & 42086 \\
\hline Renta básica anual pura (en miles) & 6 \\
\hline PIB nominal (en millones) & 1200000 \\
\hline COSTE (en millones de euros) & 252516 \\
\hline COSTE (en \% PIB) & 21,0 \\
\hline
\end{tabular}

Fuente: elaboración propia a partir de INE. Población al comienzo de 2019

En la Tabla 4 se presenta un combinado de ambos ajustes. Se excluyen como elegibles para la Renta Básica Universal tanto a los jóvenes menores de 16 años como a los extranjeros. El número total de perceptores bajaría hasta los 35 millones de ciudadanos, y el coste de esa RBU ajustada sería de unos 210.000 euros, un 17,5\% del PIB. Es decir, estamos hablando de unos ahorros de unos 71.000 euros o 6 puntos del PIB

\begin{tabular}{|l|r|}
\hline 4. RENTA BÁSICA UNIVERSAL ajuste (c) \\
\begin{tabular}{|l|r|}
\hline Población ESPAÑOLES ADULTOS (miles) & 35036 \\
\hline Renta básica anual pura (en miles) & 6 \\
\hline PIB nominal (en millones) & 1200000 \\
\hline \multicolumn{2}{|c|}{} \\
\hline COSTE (en millones de euros) & 210216 \\
\hline COSTE (en \% PIB) & 17,5 \\
\hline
\end{tabular}
\end{tabular}

Fuente: elaboración propia a partir de INE. Población al comienzo de 2019

Pero, además de los jóvenes y/o los extranjeros, podríamos excluir de la RBU a otros colectivos que perciben una renta superior a los 6.000 euros al año. Ahí ya no estaríamos hablando de una renta básica "universal", sino "condicionada". Es decir, sujeta a la condición de que el ciudadano elegible no esté percibiendo una renta superior o igual a los 6.000 euros anuales de la RB. Es decir, ni un salario, ni una pensión, ni una beca, ni unas ganancias por cuenta propia superiores a esa cantidad. Volviendo al diagrama 1, estaríamos hablando de un punto de la curva cercano al punto B. Se trataría de un coste significativamente menor, pero sujeto a un sinfín de problemas de incentivos que analizaremos en la siguiente sección. 
¿Cómo quedaría, para el caso español, el coste de esta "Renta Básica condicionada"? Habría que revisar el número de perceptores de la forma siguiente:

(i) Unos 9,2 millones de pensionistas que cobran más 500 euros al mes. Su exclusión no contradice estrictamente el carácter "universal" de la renta básica porque todos los pensionistas cobrarían, al menos, los 6.000 euros. Pero sí que atacaría su carácter incondicional, que la hacía atractiva. Habrá pensionistas que hayan contribuido al sistema a lo largo de su vida laboral y cobren, pongamos por caso, 6.500 euros al año, que se confrontarán con otros que, habiendo cotizado poco o nada, cobren los 6.000 de la renta básica, es decir, prácticamente lo mismo. Además de esos 9,2 millones, habría otros pensionistas, unos 400.000, que reciben una pensión media de 4.800 euros. En esos casos sólo habría que complementarles con 1.200 euros, hasta alcanzar los 6.000 euros de la renta básica.

(ii) A los parados, que reciben una prestación media de 3.000 euros, sólo habría que compensarles con otros 3.000. Se plantea un problema de discontinuidad semejante al del caso anterior

(iii) Los "autónomos" que declaren percibir más de 6.000 euros serían excluidos y al resto sólo se les compensaría por la diferencia; hasta llegar a los 6.00 euros

(iv) Finalmente, con los "asalariados" procederíamos de una forma equivalente: excluiríamos a los 13,6 millones que declaran ganar más de 6.000 euros, compensando al resto.

En la Tabla 5 se recoge el resumen de los cálculos del coste inicial de implantar la "Renta Básica Condicionada" una vez hechos los ajustes para evitar las duplicidades en las percepciones de la renta mínima universal.

\begin{tabular}{|l|r|}
\hline 5. RENTA BÁSICA CONDICIONADA \\
\hline Población ajustada (miles) & 10908 \\
\hline Renta básica anual (en miles) & 6 \\
\hline PIB nominal (en millones) & 1200000 \\
\hline & \\
\hline COSTE (en millones de euros) & 65448 \\
\hline COSTE (en \% PIB) & 5,5 \\
\hline
\end{tabular}

Fuente: elaboración propia a partir de INE y Agencia Tributaria

Frente al coste inicial, de un $23,5 \%$ del PIB, tras estos ajustes el coste de la renta básica se reduciría a la cuarta parte, un 5,5\% del PIB. En euros estaríamos hablando de unos 65.000 millones al año. Aunque se trata de una cantidad significativa, es mucho más manejable que en todas las versiones anteriores de la renta universal.

Además, esta magnitud se trataría de un límite superior del coste real, porque como veremos más adelante, también habría que excluir a los perceptores de rentas del capital, la deducción por hijo entre 16 y 25 años y se podrían ajustar otros gastos, como las ayudas a la dependencia, que ya estarían cubiertos por la RBU, o las políticas activas de empleo.

Al lector no se le escapa que la idea de "universalidad" queda aparcada con un enfoque como éste. Solo 11 millones de españoles, de una población de 47 millones, percibiría el "dividendo por la libertad". Se trata de los inactivos sin pensión o con pensión pequeña, de los ocupados y parados con salario y subsidio muy 
bajo, o de los excluidos del mercado de trabajo que no perciban ninguna renta o prestación. Es evidente, como argumentaremos al final, que un diseño de estas características reduciría la pobreza y, bajo ciertas condiciones, la desigualdad. Pero también es evidente que se generarían toda una serie de problemas de incentivos que veremos en la sección 4. Pero antes de entrar ahí, analizaremos, con herramientas microeconómicas, el coste de diferentes esquemas de renta básica condicionada, el equivalente al punto $C$ en la curva del Diagrama 1.

La cuantificación del coste de una renta básica universal "no condicionada" - tipo A - haciendo uso de los microdatos de la Encuesta de Condiciones de Vida del año 2018 arroja cifras muy similares a las mencionadas anteriormente. De esta forma, la implantación de una renta universal de $430 €$ ( $80 \%$ del indicador público de renta de efectos múltiples, IPREM) para toda la población supondría un coste de 237,084 millones de euros, es decir, el 19,6\% del PIB (véase escenario 1 del cuadro 6). Si se limitase el importe que perciben los niños dependientes económicamente del hogar7 al 24\% del IPREM (escenario 2 del cuadro 6) el coste disminuiría en 34,000 millones, hasta alcanzar un importe de 202,868 . Por último, si los menores dependientes no percibieran ninguna prestación el coste total sería de 188,203 millones de euros (escenario 3 del cuadro 6).

\section{Coste Renta Básica Universal a partir de la Encuesta de Condiciones de Vida 2018}

\begin{tabular}{|c|c|c|c|}
\hline ESCENARIOS & $\begin{array}{c}\text { RENTA BÁSIC } \\
\text { MENSUAL. } \\
€ / \text { mes }\end{array}$ & & COSTE \\
\hline & ADULTOS & NIÑOS & (millones de euros) \\
\hline Escenario 1 & 430 & 430 & 237,084 \\
\hline Escenario 2 & 430 & 129 & 202,868 \\
\hline Escenario 3 & 430 & 0 & 188,203 \\
\hline
\end{tabular}

Fuente: Elaboración propia a partir de la Encuesta de Condiciones de Vida 2018 Nota: 430 euros corresponde al 80\% del IPREM. 129 al 24\% del IPREM

Pero el establecimiento de una RBU podría venir acompañado, con la finalidad de evitar duplicidades, de la reformulación del sistema de prestaciones asistenciales no contributivas y de algunas de las deducciones y reducciones fiscales existentes, lo que supondría movernos del punto $A$ al punto $C$ en la curva de costes e incentivos mencionada anteriormente. La supresión de algunos de estos sistemas de protección junto con el incremento en la recaudación fiscal aparejado al aumento de renta generado por la RBU supondría potenciales ahorros del sistema que contribuirían a financiar, en parte, el elevado coste de la RBU.

En particular, el sistema de prestaciones no contributivas en España puede resumirse en cuatro grandes bloques $^{8}$. En primer lugar, con un importe aproximado de 8500 millones de euros en el año 2018, se encuentran las prestaciones destinadas a garantizar un nivel de renta mínima de las personas mayores, entre las que destaca las pensiones no contributivas y el complemento de mínimos de las pensiones, financiado con cargo a los Presupuestos Generales del Estado.

\footnotetext{
${ }^{7}$ Hijos mejores de 18 años y aquellos entre 18 y 25 años dependientes económicamente del hogar.

${ }^{8}$ Véase Estudio sobre Programas de Rentas Mínimas en España (AIREF-2019) para una profunda revisión de los sistemas de prestaciones sociales en España y en especial de los programas de rentas mínimas existentes.
} 
El segundo gran bloque de prestaciones asistenciales no contributivas son las destinadas a los desempleados que comprende el subsidio asistencial al desempleo, el subsidio de los trabajadores eventuales agrarios y los programas de protección extraordinarios desarrollados a raíz de la última crisis económica (programas PRODI, PREPARA y, desde 2015, el Programa de Activación de Empleo). Todos ellos sumaban un gasto en el año 2018 próximo a los 6000 millones de euros.

En tercer lugar, se encuentras las prestaciones económicas destinadas a la promoción de la autonomía personal, la atención y protección a las personas en situación de dependencia que contribuye a la mejora de las condiciones de vida de los ciudadanos cuyo coste en el año 2018 superó los 7400 millones de Euros.

Por último, el cuarto grupo de prestaciones son los sistemas de rentas mínimas creados por la CCAA como resultado de las competencias que en esta materia les atribuye la Constitución a los Gobiernos Regionales. Con un elevado grado de heterogeneidad, tanto en los criterios para ser beneficiarios como en los importes mínimos garantizados y en ausencia de mecanismos de coordinación interterritorial, suponen un coste global aproximado de 1500 millones de euros. Por lo tanto, los ahorros totales estimados de la supresión de las prestaciones no contributivas ascenderían a uno 18000 millones de euros, muy lejos aún del elevado coste que supone la RBU.

\section{Ahorro por eliminación de Prestaciones No Contributivas ${ }^{9}$}

\begin{tabular}{|l|l|}
\hline PRESTACIONES NO CONTRIBUTIVAS & \multicolumn{2}{|c|}{ AHORRO } \\
\hline PENSIONES NO CONTRIBUTIVAS & 8.506 \\
\hline Complemento PGE pensiones mínimas & 7.180 \\
\hline Jubilación (no incluye invalidez) & 1.326 \\
\hline DESEMPLEO & 5.966 \\
\hline Subsidio Paro y agrario & 4.920 \\
\hline Renta Activa de Inserción + Programa activación empleo & 1.046 \\
\hline DEPENDENCIA & 7.460 \\
\hline \multicolumn{2}{|l}{} \\
\hline RENTAS MÍNIMAS CCAA & 1.540 \\
\hline
\end{tabular}

Fuente: Elaboración propia a partir del Anuario de Estadistica Ministerio de Trabajo, Migraciones y Seguridad Social 2018 (SEEPROS) y PGE

A su vez, el establecimiento de una RBU contribuiría a aumentar la recaudación del IRPF tanto por el incremento de renta que supone como por la eliminación de algunas de las reducciones y deducciones fiscales existentes que pretenden no gravar importes de rentas destinados a cubrir necesidades básicas personales y familiares al igual que la RBU.

\footnotetext{
${ }^{9}$ Excluimos del análisis las prestaciones por enfermedad o invalidez y las prestaciones por hijo a cargo ya que tienen por objeto complementar el nivel de renta ante una situación de vulnerabilidad adicional sobrevenida. En cualquier caso, el coste total de ambas partidas en 2018 ascendió a 2000 millones aproximadamente.
} 
Para el cálculo del aumento de recaudación, desarrollamos un modelo que micro simulación fiscal utilizando los 2,7 millones de declaraciones de la renta de la muestra de declarantes de la Agencia Tributaria y el Instituto de Estudios Fiscales (representativa de los 19,6 millones de declaraciones existentes). Procedemos de forma que recalculamos la declaración de la renta completa para cada uno de los contribuyentes como si hubieran experimentado un incremento de su renta de 430 euros brutos mensuales y obtenemos el resultado final de su declaración. De esta forma, el avance de la recaudación por IRPF como consecuencia de la RBU sería cercano a los 23.000 millones de euros ${ }^{10}$.

Pero, además, el avance de la recaudación sería de mayor magnitud si eliminásemos aquellas deducciones y reducciones fiscales que persiguen objetivos similares a la renta básica universal. En particular, la eliminación del mínimo personal y familiar, cuya finalidad es no someter a tributación importes de rentas destinadas a cubrir las necesidades básicas personales y familiares del contribuyente supondría ahorros estimados por un importe cercano a los 19.000 millones de euros. Por último, si estimamos el impacto fiscal conjunto del aumento de renta junto con la eliminación del mínimo personal y familiar, los ahorros alcanzarían la cifra de 45.000 millones de euros.

\section{Ahorro por incrementos de recaudación (IRPF) y eliminación de Deducciones y Reducciones Fiscales}

\begin{tabular}{|l|r|}
\hline EFECTO DE LA RENTA BÁSICA SOBRE EL IRPF & $\begin{array}{c}\text { AHORRO } \\
\text { (millones de euros) }\end{array}$ \\
\hline Incremento recaudación por implantación de la renta básica & 22974 \\
\hline Eliminación del Mínimo Personal y Familiar & 19060 \\
\hline Incremento Renta y Eliminación deducciones (efecto conjunto) & 44987 \\
\hline
\end{tabular}

Fuente: Microsimulación propia a partir de los microdatos de la muestra de declarates de la Agencia Tributaria e Instituto de Estudios Fiscales 2016. Nota: Para la elaboración de esta tabla recalculamos, en primer lugar, la declaración de la renta para cada uno los 2,7 millones de declarantes de la muestra de declarantes de la Agencia Tributaria (representativa de los 19,6 millones de declarantes) suponiendo un aumento de la renta bruta mensual de 430 euros. En segundo lugar, volvemos a calcular la declaración para cada declarante suponiendo que se elimina el mínimo personal y familiar. Finalmente la cifra de 52148 se obtiene como resultado de incrementar la renta bruta de cada declarante en 430 euros mensualmente y de eliminar el mínimo personal y familiar de forma simultanea.

Por último, el establecimiento de una renta básica universal nos podría llevar a reflexionar sobre una posible modificación del sistema de prestaciones contributivas existentes. En este sentido, aunque la provisión de una renta mensual garantizada permitiría disminuir los importes de las pensiones contributivas o de las prestaciones por desempleo también debería venir acompañada de una disminución de las cotizaciones sociales con las que se financian, por los que los potenciales ahorros dependerían de la reforma específica que sobre ellas se llevasen a cabo (véase cuadro 9 para información de los gastos e ingresos de las prestaciones contributivas en la actualidad).

\footnotetext{
${ }^{10}$ El aumento de renta derivado de la RBU podría suponer también un avance de la recaudación del impuesto sobre el valor añadido al trasladarse una parte del incremento de renta al consumo. En cambio, la heterogénea elasticidad renta-consumo de los hogares con diferente nivel de renta y riqueza hace difícil una estimación precisa de dicho importe.
} 
9. Resumen de gastos e ingresos del sistema de prestaciones contributivas en la actualidad

\begin{tabular}{|c|c|c|}
\hline PRESTACIONES CONTRIBUTIVAS & $\begin{array}{c}\text { GASTOS } \\
\text { (millones de euros) }\end{array}$ & $\begin{array}{l}\text { INGRESOS } \\
\text { (millones de euros) }\end{array}$ \\
\hline DESEMPLEO & 10849 & \\
\hline PENSIONES CONTRIBUTIVAS & 122277 & \\
\hline COTIZACIONES SOCIALES & & 110560 \\
\hline
\end{tabular}

Fuente: Anuario de Estadistica Ministerio de Empleo y Seguridad Social (SEEPROS) y PGE

Como conclusión, los resultados mostrados en esta sección evidencian de una forma muy clara que los costes brutos de la renta básica universal incondicional de tipo A son muy elevados y cercanos a los 200,000 millones de euros en sus diferentes configuraciones (16,5\% del PIB). Los potenciales ahorros derivados de movernos desde $A$ hasta $C$ permitirían financiar entre un 40 y un $50 \%$ del coste total de la misma, siendo el coste de la RBU aún muy elevado. Por su parte, pasar del punto A al B supondría renunciar al carácter universal de la medida en favor de figuras alternativas como pueden ser el impuesto negativo, la renta mínima o el complemento salarial cuyo coste económico es mucho más reducido pero sus problemas de incentivos mucho mayores. Pero antes de abordar una transformación de tal envergadura, merece la pena tener claro los importantes problemas en términos de incentivos que genera la implantación de una Renta Básica Universal.

\section{EL PROBLEMA DE INCENTIVOS}

La Renta Básica Universal, como tal, nunca se ha implantado. Como se ha mencionado anteriormente, se han realizado experimentos en Finlandia y en Holanda, pero referida a grupos de perceptores concretos, sin carácter universal. Por tanto, hasta la fecha, todo lo que podamos decir sobre los posibles incentivos negativos que pueda ocasionar tiene, por tanto, un carácter teórico o académico. Entre los incentivos podemos destacar:

- (i) Una reducción de la oferta efectiva de trabajo

- (ii) Desempleo estructural, al elevarse el "salario de reserva"

- (iii) Desactivación de parte de la población activa

- (iv) Menor ahorro, si la garantía de una renta de supervivencia de por vida reduce el incentivo a ahorrar.

- (v) Menor acumulación de capital humano, si percibir una RBU supone un incentivo a abandonar los procesos de formación.

- (vi) Más economía sumergida, al ser la RBU incompatible con otras fuentes de ingreso, incentivaría sumergir actividades en el sector servicios que sean difícilmente fiscalizables. 
Probablemente, el argumento que más preocupa a los economistas es el primero, que está estrechamente ligado al segundo. En el Diagrama 2 presentamos, utilizando un modelo estándar de mercado de trabajo no competitivo, como por ejemplo, salarios de eficiencia"1, el impacto teórico de la introducción de alguna forma de renta básica. La curva creciente LS, una relación de fijación de salarios semejante a la oferta efectiva de trabajo parte de un nivel llamado salario de reserva, por debajo del cual no se acepta trabajar ninguna cantidad de horas.

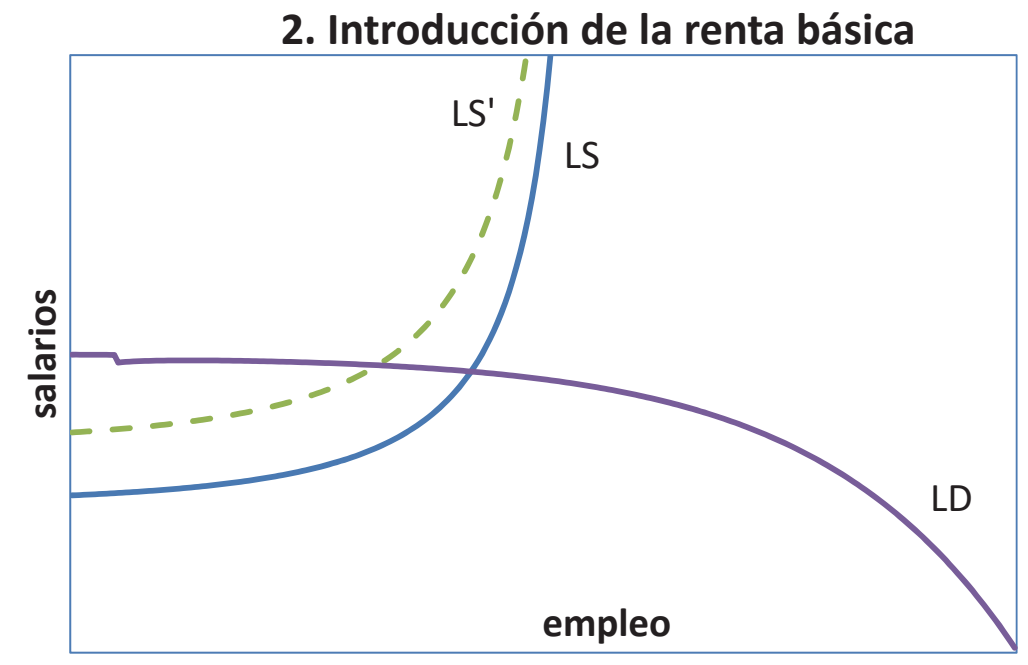

Por otro lado, habría una demanda de empleo, que llevan a cabo las empresas, representada por la curva decreciente LD. La introducción de la renta básica equivale a un aumento del salario de reserva, lo que desplaza la curva de salarios a la izquierda hasta LS'. Nótese que, para niveles de salarios altos, el impacto de la renta básica sobre la curva de oferta es prácticamente irrelevante. Es decir, el desplazamiento de la curva de oferta de trabajo efectiva o curva de salarios no es paralela.

El resultado de la introducción de la renta básica es que aumenta el salario de equilibrio y se reduce el empleo. En este Diagrama 2 se ha presentado un caso en que la curva de demanda de trabajo que realizan las empresas es muy elástica. Por este motivo, la subida del salario no es muy grande, pero la destrucción del empleo es elevada. Podría haberse planteado una situación en la que la demanda de empleo LD es más vertical (rígida). En ese caso, el aumento del salario sería mayor y la destrucción de empleo menor. Nótese además que si esta "oferta efectiva de trabajo" es distinta de la oferta nocional o población activa, habría un nivel de paro estructural en la economía, y la introducción de la renta básica elevará dicho paro estructural, tal y como señala el problema de incentivos (ii).

El problema (iii) estaría relacionado con los dos anteriores. No hablaríamos tanto de ejercer una mayor presión salarial sobre los ocupados, por parte de los trabajadores y de los empresarios, como de un abandono de la población activa o "desactivación". Aunque ello podría mejorar la tasa de paro a corto plazo, se trataría de una menor tasa de empleo a largo plazo ${ }^{12}$, así como una disminución de la renta per cápita media del país y un empeoramiento de los ratios de sostenibilidad del Estado del Bienestar (pensiones, salud, dependencia).

${ }^{11}$ Véase, por ejemplo, Sorensen y Witta-Jacobsen (2011)

${ }^{12}$ Recordemos que la tasa de empleo es igual a la tasa de actividad multiplicada por (1-tasa de paro) 
El problema de incentivos (iv) sobre el ahorro es más discutible. Si partimos de un modelo en el que la tasa de ahorro de las familias depende de la incertidumbre sobre las rentas futuras, es un contexto de maximización de la utilidad del consumidor a lo largo de toda su vida, una reducción de dicha incertidumbre, al disponerse de una fuente garantizada de renta, podría reducir la tasa de ahorro y, por tanto, la tasa de inversión y el crecimiento a largo plazo de la economía.

Relacionado con la desactivación y el menor incentivo a ahorrar se encuentra el problema ( $v$ ), es decir, un menor incentivo por parte del trabajador, el parado o el inactivo a llevar a cabo programas de formación, avanzar en sus niveles de estudios o simplemente profundizar en sus conocimientos. En ese caso se produciría una menor acumulación de capital humano. Tanto (iv) como (v) se traducirían en un menor crecimiento de la Productividad Total de los Factores (PTF), que es el motor de crecimiento económico a largo plazo.

Finalmente, el problema (vi) es menos discutible. Si la RBU es incompatible con otras fuentes de renta y el perceptor la considera insuficiente para mantener un nivel deseado de consumo, tanto las empresas como los perceptores tendrán un incentivo a contratar "en negro" con el consiguiente aumento de la economía sumergida y el coste fiscal asociado a ella. Este incentivo será mayor especialmente en el sector servicios, en donde la fiscalización de la actividad económica es más difícil. Y, por supuesto, el incentivo será mayor cuanto mayor sea la condicionalidad de la RBU y, por tanto, la incompatibilidad. Con una renta básica genuinamente universal, sin incompatibilidades de ningún tipo, desaparecería este incentivo a sumergir la actividad económica.

\section{LA POBREZA Y LA DESIGUALDAD}

No queremos terminar este recorrido crítico sobre la renta básica sin hacer mención al posible impacto sobre la pobreza y la desigualdad que, tal y como mencionábamos en la introducción, es una de las razones que más está impulsando la apuesta por la renta básica. Parece razonable pensar que la RBU mejorará la pobreza, al elevar la renta de los ciudadanos por debajo del umbral de pobreza, al menos en un enfoque estático. En un enfoque dinámico habría que tener en cuenta los efectos permanentes sobre el crecimiento a largo plazo.

El impacto sobre la desigualdad es, sin embargo, más discutible. Consideremos el caso descrito en el Diagrama 2 en el que el salario medio apenas aumenta, aunque se destruya empleo. En ese caso, es plausible que la desigualdad de rentas disminuya. Sin embargo, en caso de que la demanda de empleo fuera más rígida, la subida salarial como consecuencia de la introducción de la renta básica de los que mantienen su empleo sería muy superior. En ese caso tendríamos a los ocupados, con una renta más elevada y a los perceptores de subsidio de desempleo y los inactivos, con una renta similar al de la prestación básica. En ese caso, la desigualdad de renta podría empeorar. Si, además, consideramos una oferta y demanda de trabajo diferenciada entre diferentes sectores o niveles de cualificación, podríamos encontrar casos en los que la RBU eleve más el salario de reserva y la presión salarial de los más cualificados, pues estos tendrían una demanda de empleo más rígida (vertical). Por el contrario, los trabajadores con menos cualificación tendrían una demanda de empleo más elástica (horizontal) y el impacto sobre el salario sería menor. En este caso, el aumento de la desigualdad no sólo se produciría entre trabajadores con empleo y perceptores de la renta básica, sino también dentro del grupo de los trabajadores ocupados, para los que empeoraría la dispersión salarial. 
A medio y largo plazo la RBU puede, además, empeorar la distribución de la renta por otros varios motivos:

(i) Si se abandonan las políticas de formación para los perceptores de una RBU permanente, que les garantizará un nivel de vida digno, pero no les hará partícipe de los aumentos de la productividad del conjunto de la economía, el crecimiento económico empeorará la desigualdad: los preceptores de la RBU sólo verán cubierto su poder adquisitivo con subidas del IPC, en el mejor de los casos. Los trabajadores ocupados tendrán subidas salariales que cubran tanto el IPC como el aumento de la productividad ${ }^{13}$

(ii) Las empresas podrían incentivos a desprenderse de los trabajadores menos eficientes, que ya están cubiertos por la RB, al sentir menor presión social y sindical para mantenerlos en sus puestos de trabajo.

(iii) El coste fiscal de la RBU puede provocar recortes en otras políticas que garantizan la igualdad de oportunidades, como son la Educación o la Sanidad, empeorando la distribución de la renta a largo plazo. Tal y como se señaló en la introducción, este fue el principal motivo de rechazo de la RBU en algunos países, como Holanda, en los años 80.

\section{CONCLUSIONES}

En este trabajo hemos repasado varias definiciones de renta básica y hemos analizado sus problemas de costes e incentivos y la relación que existe entre ambos. En general, concluimos que hay una relación inversa entre ambos: cuanto más incondicional sea la renta básica, menos problemas de incentivos presentará, aunque tendrá un mayor coste.

Hemos estimado, para el caso español el coste de implantación de diversas modalidades de renta básica, en función de su condicionalidad, utilizando tanto un enfoque macroeconómico como microeconómico. Los resultados de ambos enfoques son similares y nos llevan a concluir que el coste de la RBU incondicional es inasumible, lo que requiere introducir restricciones en el uso de la renta básica, limitando el número de beneficiarios y la cuantía percibida en función de la situación laboral y económica de los potenciales perceptores. Y esto nos lleva al problema de incentivos, que sólo pueden ser abordados desde un punto de vista teórico, pero que pueden tener impactos relevantes sobre la sostenibilidad del Estado del Bienestar. Implantar la renta básica de modo permanente requiere de una estimación más fina de sus implicaciones a largo plazo, lo que sólo puede ser abordado con una mayor profundización avanzando en el análisis experimental.

${ }^{13} \mathrm{~A}$ largo plazo los salarios reales crecen con la productividad del trabajo 


\section{REFERENCIAS BIBLIOGRÁFICAS}

- AIREF (2019) "Los Programas de Rentas Mínimas en España”.

- COLLECTIF CHARLES FOURIER (1985), "L'allocation universelle”, La Revue Nouvelle 4

- INE (2019a) "Encuesta Continua de Hogares".

- INE (2019b) "Encuesta de Población Activa”.

- SORENSEN, P.B. y H.J. WHITTA-JACOBSEN, H.J. (2011): Introducing Advanced Macroeconomics, McGrawHill (2n edition).

- TORRES LÓPEZ, J. (2019), “La Renta Básica”, ediciones Deusto. 